\title{
SEA ICE DETECTION USING GNSS-R DATA FROM UK TDS-1
}

\author{
A. Alonso-Arroyo ${ }^{1,2}$, V. U. Zavorotny ${ }^{1}$, and A. Camps $^{2}$ \\ ${ }^{1}$ Earth System Research Laboratory, NOAA, Boulder CO, 80305 U.S.A. \\ ${ }^{2}$ Universitat Politècnica de Catalunya - BarcelonaTech and IEEC/CTE-UPC
}

\begin{abstract}
This work demonstrates a methodology to detect sea ice presence over the Arctic and Antarctic regions using Global Navigation Satellite Systems (GNSS)-Reflectometry (GNSSR) data obtained with the UK TDS-1 satellite. The algorithm is based on estimating the degree of coherence of the received GNSS reflected waveform or Delay-Doppler Map (DDM). While at open ocean conditions, the scattered signal follows the diffuse scattering model, over flat sea ice it follows the coherent scattering model. In order to measure the degree of coherence of the received waveform or DDM, a correlation with the clean Woodward Ambiguity Function (WAF) is performed. The more similar the received signal is to the $\mathrm{WAF}$, the more coherent is the scattering, and consequently, the more likely a flat sea ice surface is involved. In order to assess the performance of the proposed estimator a probabilistic study based on a Bayesian approach is performed, using the OSISAF Sea Ice Concentration (SIC) maps as ground truth. A probability of detection of $97 \%$, a probability of false alarm of $2 \%$, and a probability of error of $2.5 \%$ are the best results obtained for the Arctic region.
\end{abstract}

Index Terms - Sea Ice, GNSS-R, UK TDS-1, coherent reflection, incoherent scattering.-

\section{INTRODUCTION}

The detection and monitoring of sea ice has been performed using active and passive techniques. Active techniques based on real aperture radar and synthetic aperture radar (SAR) generally measure surface roughness, which leads to sea ice type classification, since the waveform shape is highly sensitive to surface roughness [1]. However, in order to achieve a high resolution, the frequency band used is normally Ku-band or K-band (12-18 GHz and 18-26 GHz respectively), which makes the radar technique very sensitive to both small and large scale roughness. Passive techniques based on microwave radiometry have been used to determine the Sea Ice Concentration (SIC) parameter, which is the percentage of ice in a pixel [2]. For instance $0 \%$ indicates open water, $50 \%$ indicates that half of the pixel is covered by ice, and $100 \%$ indicates that the entire pixel is solid ice. In order to achieve a high resolution, very high frequencies are used in radiometric systems, such as the $90 \mathrm{GHz}$ band of the SSMIS or the AMSR2 radiometers.
Multistatic techniques using Global Navigation Satellite System (GNSS) signals of opportunity for remote sensing were discussed in the late 80s [3]. In 1993, it was proposed to use those signals as an alternative to radar altimeters for the measurement of mesoscale altimetry, also known as the PAssive Reflectometry and Interferometry System (PARIS) concept [4]. In 1994, a publication reporting an incident that occurred in 1991 with a French military aircraft getting locked to the Global Positioning System (GPS) signal reflected from the ocean surface, presented an evidence that such a signal can be detected on board an airplane [5]. In 2000 , initial results comparing the waveform peak power of the GNSS reflected signals over ice against RADARSAT back-scattering echoes were presented [6]. In 2003, a theoretical model explaining the sea ice scattering was proposed [7]. In 2006, it was shown that there is a strong presence of the coherent component in the GNSS-R bistatic scattering echoes [8], indicating a deficiency of a purely diffusive scattering model for such cases. This was confirmed in 2010 with a detailed study using United Kingdom Disaster Monitoring Constellation (UK-DMC) GNSS-R dataset [9]. However, unambiguous relations between waveform peak power or shape and sea ice parameters have not been found. Airborne studies using GNSS-R data were also performed in 2010 for the determination of sea ice parameters [10]. Also, the effect of surface roughness was analyzed and compared to lidar measurements in that publication.

In all previous publications, the presence of a coherent component in the sea ice scattered signal was found. This work uses the reflected signal coherence to detect the presence of a planar sea ice and determine whether the scattering comes from open water or from sea ice. Also, a statistical analysis is performed allowing to compare the results from the estimator with the Ocean and Sea Ice (OSI) SAF Sea Ice Concentration (SIC) maps.

\section{THEORETICAL BACKGROUND}

The sea ice scattering process in a forward scattering configuration was studied long ago from a nadir geometrical configuration, which is the one found in conventional satellite radar altimetry. The power waveform or returned power as a function of the delay $(\tau)$ is composed of three distinct components [11]-[13]:

$$
W(\tau)=S_{r}(t) * P_{F S}(t) * r(t)
$$


where $S_{r}(t)$ is the shape of the transmitted pulse or point target response, $P_{F S}(t)$ is the flat surface response, which is the radar cross section as a function of the delay time weighted by the antenna gain pattern, and $r(t)$ characterizes the surface roughness $r m s$, and it is the mean surface density of point scatterers as a function of the delay time.

In the case of conventional GNSS-R (cGNSS-R) for the public C/A signal, the equivalent radar pulse length is 293 meters (1 chip). This means that, unless there is a significant variation of the surface heights, the term $r(t)$ does not affect severely the waveform shape, since the addition of the different echoes from each scatterer occurs within the same chip. Consequently, Eqn. (1) becomes:

$$
W(\tau) \approx S_{r}(t) * P_{F S}(t)
$$

Note that the nadir-looking observation is a particular case of the forward scattering GNSS-R geometry. In 2000, a theoretical scattering model was proposed based on the Geometric Optics (GO) limit of the Kirchhoff Approximation (KA) to explain the shape of the GNSS ocean scattered signals [14]. This model was mathematically reformulated later as follows [15]:

$$
\operatorname{DDM}(\tau, f)=|\chi(\tau, f)|^{2} * * \sigma^{0}(\tau, f)
$$

where DDM is the Delay-Doppler Map, $|\chi(\tau, f)|^{2}$ is the WAF, and $\sigma^{0}(\tau, f)$ is the radar cross section including the antenna gain pattern projection.

Equation (3) can be used only when the surface is sufficiently rough. It describes how the power is spread in the Delay-Doppler domain. For example, for open water, due to wind-generated waves the spreading of the power leads to the "horseshoe" shape for the DDM [8]. The transition to an almost flat surface cannot be done within Eqn. (3). This equation does not describe a coherent field which is the result of a mirror-like reflection from such a surface. Use of the slope probability function in Eqn. (3) in the form of a deltafunction is intuitive, but not legitimate. It will lead to a wrong result. The coherent form of the DDM should be based on the original Kirchhoff approximation for the scattered field under the assumption that the surface roughness is very small (the Rayleigh parameter is less than 1). More details on the modeling of the coherent component of the microwave signal reflected from a flat ice surface can be found in [16].

\section{UK TDS-1 AND GROUND-TRUTH}

\subsection{UK TDS-1}

The United Kingdom TechDemoSat-1 (UK TDS-1) mission is the natural continuation of the United Kingdom Disaster Monitoring Constellation (UK-DMC) mission. The satellite orbit is approximately $630 \mathrm{~km}$ in altitude, and apart from several payloads, it contains a receiver of GNSS signal reflections. This payload is the natural evolution of the one used in the pioneering UK-DMC experiment. It is based on a down-looking patch $2 \times 2$ antenna array and a sampler with real-time processing capabilities. The GNSS-R payload is capable to sample data and record it, or process it on-board too. The real-time processing is based on generating DDMs with $1 \mathrm{~ms}$ coherent integration time and 1000 incoherent summations. This leads to the L1b product, saving significant time and resources for the data to be downloaded. The DDMs generated are windowed for 128 Delay bins with 244 ns resolution, and 20 Doppler bins with $500 \mathrm{~Hz}$ resolution. All the L1b data available from the Measurement of Earth Reflected Radio-navigation Signals By Satellite (MERRByS) server have been used for this work [17].

\subsection{OSI SAF Data}

The Ocean and Sea Ice (OSI) SAF project bears on an European consortium hosted by Méteo-France [18]. It provides daily Sea Ice Concentration (SIC) maps, Sea Ice Edge (SIE) maps, and Sea Ice Type (SIT) maps from both Arctic and Antarctic regions. Those maps are generated from a combination of the 19,37 , and $91 \mathrm{GHz}$ channels from the passive microwave radiometer of the SSMIS sensor. In the case of the SIE and SIT maps, also ASCAT data is used in the retrieval algorithm [19]. The higher resolution is achieved by the $91 \mathrm{GHz}$ channel $(12.5 \mathrm{~km} \times 12.5 \mathrm{~km})$, and the other channels are used for atmospheric compensation and data quality assessment. The maps shown in this work have been obtained from the OSISAF server [18].

\section{DATA PROCESSING AND SEA ICE MAPS}

The L1b data from the UK TDS-1 mission are uncalibrated because there is neither calibration with the direct signal nor with the system parameters, so the only direct observable obtained is the Signal-to-Noise Ratio (SNR). In order to avoid calibration issues, all DDMs have been normalized. Apart from that, since the power can be spread in the Delay-Doppler domain due to the surface scattering, the DDMs have been integrated along the Doppler domains in order to obtain the equivalent waveform that accounts for all delays, as it is the case of the nadir satellite altimetry. To measure the degree of coherency the correlation operation has been used, since it measures the degree of similarity between the two signals being correlated. A result close to 1 means that they are practically equal, whereas a result close to 0 means that they are totally different. In order to apply the correlation operation, the normalized waveforms are scaled again to obtain energy-unit signals, so the correlation operation result is always between 0 and 1 . In this case, the Doppler integrated waveforms obtained from the UK TDS-1 have been correlated against the Doppler cut of the WAF of the satellite C/A signal currently analyzed. Figure 1 shows two SIC maps, for both Arctic and Antarctic regions, with the correlation results overlapped for the different reflected signals picked on February $4^{\text {th }} 2015$. 
Therein, when there is ice presence the correlation result tends to 1 and for open water it tends to 0.35 .

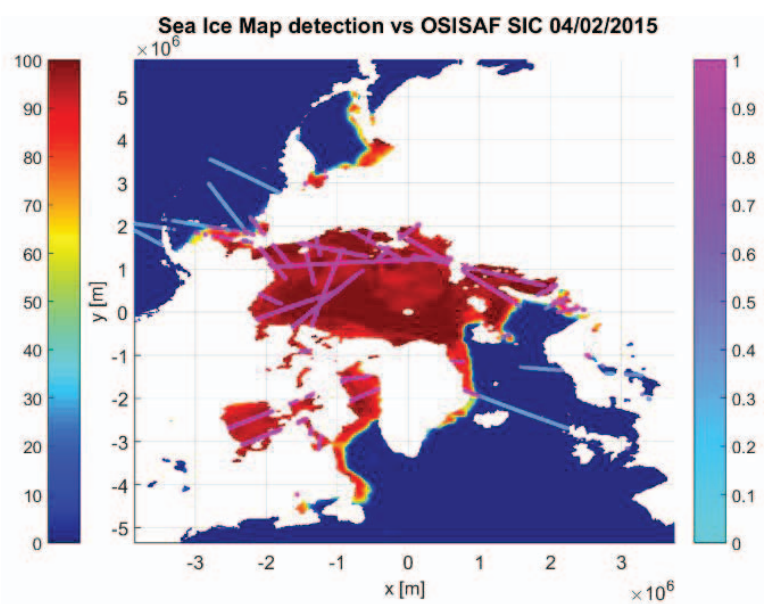

(a)

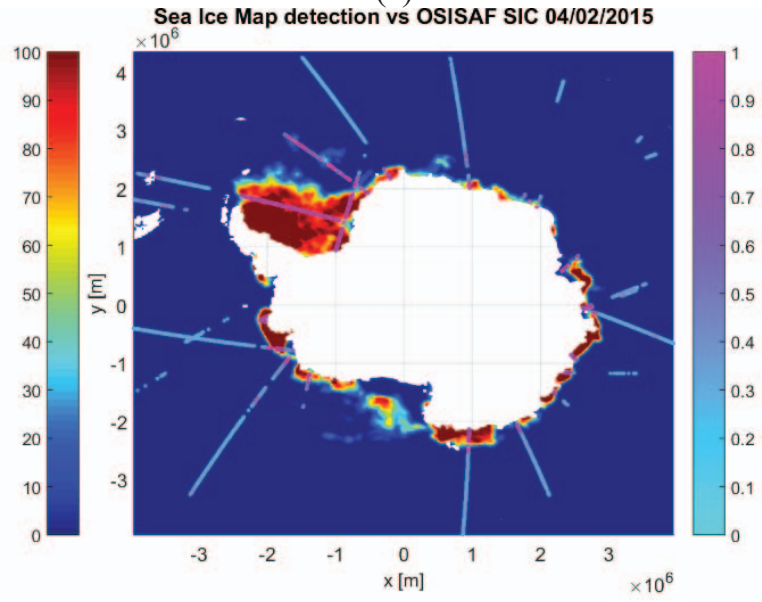

(b)

Figure 1: SIC maps against correlation results on February 4th for the (a) Arctic region, (b) Antarctic region.

A Bayesian approach is followed in order to assess the performance of the estimator proposed and evaluate it objectively [20]. From the entire dataset available at the UK TDS-1 website, the probability of detection $(\mathrm{Pd})$, the probability of false alarm (Pfa), the probability of error $(\mathrm{Pe})$, and the threshold are computed in order to determine if the surface under observation is sea ice or open. Figure 2 shows the $p d f$ s computed for the sea ice and the open water for both Arctic and Antarctic regions.

Table 1 shows the performance of the estimator proposed. Note that even though the threshold is not the same for both cases, due to the center value and width of $p d f \mathrm{~s}$ shown in Fig. 2, small variations of the threshold will not change the performance of the estimators since the points are concentrated around 0.3 for the open water case and around 0.95 for the sea ice case. Furthermore, the distance between $p d f \mathrm{~s}$ is much larger than their corresponding width in both Arctic and Antarctic cases.

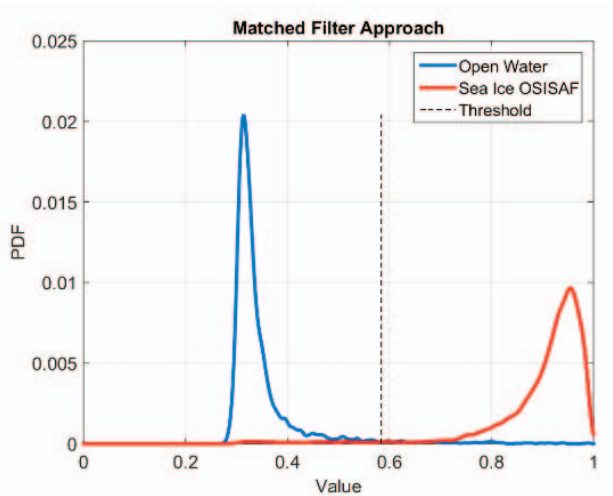

(a)

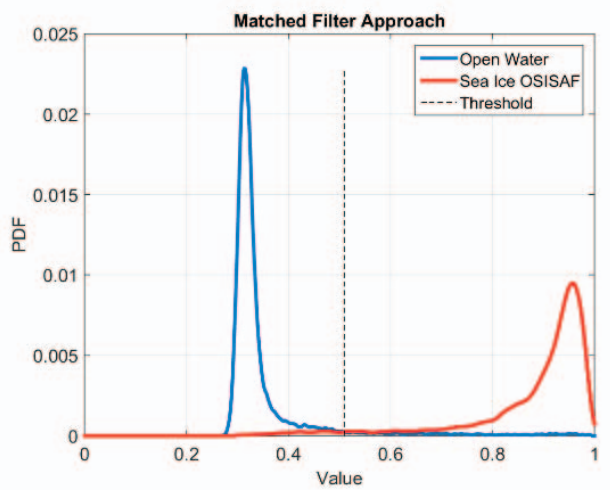

(b)

Figure 2: Estimator performance for the (a) Artic region, (b) Antarctic region.

Table 1: Performance evaluation of the estimator proposed.

\begin{tabular}{|c|c|c|c|c|}
\hline & $\mathrm{Pd}$ & Pfa & $\mathrm{Pe}$ & Threshold \\
\hline Arctic & 0.974 & 0.023 & 0.025 & 0.583 \\
\hline Antarctic & 0.963 & 0.054 & 0.045 & 0.510 \\
\hline
\end{tabular}

Since the reflection from sea ice follows the coherent model, the ground resolution corresponds to the first Fresnel zone [21]. For the UK TDS-1 conditions, taking into account the platform speed and the integration time, it corresponds to an area of approximately $6 \mathrm{~km} \times 0.4 \mathrm{~km}$ size, in the alongtrack and across-track directions respectively. This is even a better resolution than the one obtained with the $90 \mathrm{GHz}$ band of current microwave radiometers.

\section{CONCLUSIONS}

This work has demonstrated a simple methodology to detect sea ice presence over the ocean for the Polar Regions (Arctic and Antarctic). The $1 \mathrm{~ms}$ coherently integrated and 1000 incoherently summed DDMs from the UK TDS-1 mission have been also integrated along the Doppler domain in order to obtain the bistatic scattered power as a function of the delay. Then, they have been normalized first to its maximum value and then to its energy. In order to verify the similarity of the received waveform to the coherent ones, they have been correlated against the clean replicas of their 
corresponding WAF, and the result compared against the SIC maps from the OSISAF dataset. The performance of this estimator has been evaluated for the entire UK TDS-1 dataset available, obtaining a $\mathrm{Pd}$ of $97.4 \%$ for the Arctic region and a $\mathrm{Pd}$ of $96.3 \%$ for the Antarctic region. The $\mathrm{Pfa}$ is $2.3 \%$ for the Arctic region and $5.4 \%$ for the Antarctic region. The probability of error is $2.5 \%$ for the Arctic region and $4.5 \%$ for the Antarctic region. A threshold value between 0.5 and 0.6 does not change the performance of the detection.

\section{ACKNOWLEDGMENTS}

The sea ice concentration product from the EUMETSAT OSI SAF. Ice concentration is computed from atmospherically corrected SSMIS brightness temperatures, using a combination of state-of-the-art algorithms. It is operational since 2005. Sea ice concentration data from $1 / 09 / 2014$ to $22 / 02 / 2015$ were obtained from http://osisaf.met.no/p/ice/. (OSISAF SSMIS data).

The authors would like to thank SSTL and the Measurement of Earth Reflected Radio-navigation Signals By Satellite (MeRRByS) project for the UK TDS-1 data provided to conduct this research at no cost.

This work has been sponsored partly by the the project "AGORA: Técnicas Avanzadas en Teledetección Aplicada Usando Señales GNSS y Otras Señales de Oportunidad" of the Spanish Ministerio de Economía y Competitividad, Grant No ESP2015-70014-C2-1-R (MINECO/FEDER).

The authors would like to thank Scott Gleason for discussions about how to process TDS-1 data.

The authors would also like to thank Carolina Gabarro for sea ice detection discussion and for providing some processed SMOS data that could be used together with TDS1 data for Sea Ice Mapping.

Alberto Alonso Arroyo would like to thank the financial support provided by the Fulbright Commission in Spain through a Fulbright grant.

\section{REFERENCES}

[1] M. Zygmuntowska, K. Khvorostovsky, V. Helm, and S. Sandven, "Waveform classification of airborne synthetic aperture radar altimeter over Arctic sea ice," Cryosph., vol. 7, no. 4, pp. 1315-1324, Aug. 2013.

[2] G. Spreen, L. Kaleschke, and G. Heygster, "Sea ice remote sensing using AMSR-E 89-GHz channels," J. Geophys. Res., vol. 113, no. C2, p. C02S03, Jan. 2008.

[3] C. D. Hall and R. A. Cordey, "Multistatic Scatterometry," in International Geoscience and Remote Sensing Symposium, "Remote Sensing: Moving Toward the 21st Century”., 1988, pp. 561-562.

[4] M. Martín-Neira, "A passive reflectometry and interferometry system(PARIS): Application to ocean altimetry," ESA J., vol. 17, pp. 331-355, 1993.

[5] J.-C. Auber, A. Bibaut, and J.-M. Rigal, "Characterization of Multipath on Land and Sea at GPS Frequencies," in Proceedings of the 7th International Technical Meeting of the Satellite Division of The Institute of Navigation (ION GPS 1994), 1994, pp. 1155-1171.
[6] A. Komjathy, J. Maslanik, V. U. Zavorotny, P. Axelrad, and S. J. Katzberg, "Sea ice remote sensing using surface reflected GPS signals," in IGARSS 2000. IEEE 2000 International Geoscience and Remote Sensing Symposium. Taking the Pulse of the Planet: The Role of Remote Sensing in Managing the Environment. Proceedings (Cat. No.00CH37120), vol. 7, pp. 2855-2857.

[7] M. Wiehl, B. Legrésy, and R. Dietrich, "Potential of Reflected GNSS Signals for Ice Sheet Remote Sensing," Prog. Electromagn. Res., vol. 40, pp. 177-205, 2003.

[8] S. Gleason, "Remote Sensing of Ocean, Ice and Land Surfaces Using Bistatically Scattered GNSS Signals From Low Earth Orbit," University of Surrey, 2006.

[9] S. Gleason, "Towards Sea Ice Remote Sensing with Space Detected GPS Signals: Demonstration of Technical Feasibility and Initial Consistency Check Using Low Resolution Sea Ice Information," Remote Sens., vol. 2, no. 8, pp. 2017-2039, Aug. 2010.

[10] M. B. Rivas, J. A. Maslanik, and P. Axelrad, "Bistatic Scattering of GPS Signals Off Arctic Sea Ice," IEEE Trans. Geosci. Remote Sens., vol. 48, no. 3, pp. 1548-1553, Mar. 2010.

[11] G. Brown, "The average impulse response of a rough surface and its applications," IEEE Trans. Antennas Propag., vol. 25, no. 1, pp. 67-74, Jan. 1977.

[12] C. Rapley and A. P. R. Cooper, "Applications and Scientific Uses of ERS-1 Radar Altimeter Data," 1985.

[13] M. R. Drinkwater, "Ku band airborne radar altimeter observations of marginal sea ice during the 1984 Marginal Ice Zone Experiment," J. Geophys. Res., vol. 96, no. C3, p. 4555, 1991.

[14] V. U. Zavorotny and A. G. Voronovich, "Scattering of GPS signals from the ocean with wind remote sensing application," IEEE Trans. Geosci. Remote Sens., vol. 38, no. 2, pp. 951-964, Mar. 2000.

[15] T. Elfouhaily, D. R. Thompson, and L. Linstrom, "DelayDoppler analysis of bistatically reflected signals from the ocean surface: theory and application," IEEE Trans. Geosci. Remote Sens., vol. 40, no. 3, pp. 560-573, Mar. 2002.

[16] G. S. Brown, "A theory for near-normal incidence microwave scattering from first-year sea ice," Radio Sci., vol. 17, no. 1, pp. 233-243, Jan. 1982.

[17] "UK Tech-DemoSAT data." [Online]. Available: http://www.merrbys.co.uk/. [Accessed: 08-Jun-2015].

[18] "Ocean \& Sea Ice SAF: Sea Ice Concentration Maps." [Online]. Available: http://osisaf.met.no/p/ice/.

[19] S. Andersen, L.-A. Breivik, S. Eastwood, G. Oysten, T. Lavergne, M. Lind, and M. Porcires, "Ocean \& Sea Ice SAF: Sea Ice Product User's Manual, OSI-401-a, OSI-402a, OSI-403-a.," 2014.

[20] S. M. Kay, Fundamentals of Statistical Signal Processing. Volume II: Detection Theory., First Edit. Prentice Hall, 1998.

[21] J. L. Garrison and S. J. Katzberg, "Detection of ocean reflected GPS signals: theory and experiment," Proc. IEEE SOUTHEASTCON '97. "Engineering New Century," 1997. 